\title{
Pacific
}

Journal of

Mathematics

\section{ON A PRIME ZETA FUNCTION OF A GRAPH}

Takehiro Hasegawa And SeIKen Saito

Volume 273 No. 1

January 2015 


\title{
ON A PRIME ZETA FUNCTION OF A GRAPH
}

\author{
Takehiro Hasegawa And Seiken Saito
}

\begin{abstract}
In the first half of this paper, we introduce a prime zeta function associated with the Ihara zeta function, and study several properties of this function. In the last half, using results of the first half, we present graph-theoretic analogues to Mertens' theorems.
\end{abstract}

\section{Introduction}

Throughout this paper, we use the notation of [Stark and Terras 1996; Terras 2011] for graph theory and the theory of (Ihara) zeta functions $Z_{X}(u)$ of graphs, and the notation of [Hardy and Wright 2008] and [Titchmarsh 1958; 1986] for the theory of functions and the Riemann zeta function $\zeta(s)$.

In the analytic theory of the Riemann zeta function, the following theorems are well-known:

- Mertens' first theorem [1874, Equality (5)] (also see [Hardy and Wright 2008, Theorem 425], [Jameson 2003, Theorem 2.6.3], and [Titchmarsh 1986, Equality (3.14.3)]): as $x \rightarrow \infty$,

$$
\sum_{p \leq x} \frac{\log p}{p}=\log x+O(1) .
$$

- Mertens' second theorem [1874, Equality (13)] (also see [Hardy and Wright 2008, Theorem 427], [Jameson 2003, Theorem 2.6.4/Exercise 4, p. 191], and [Titchmarsh 1986, Equality (3.14.5)]): as $x \rightarrow \infty$,

$$
\sum_{p \leq x} \frac{1}{p}=\log (\log x)+B_{1}+O\left(\frac{1}{\log ^{k} x}\right)
$$

for each $k \geq 1$, where $B_{1}=0.26149 \ldots$ is the Mertens constant.

- Mertens' third theorem [1874, Equality (15)] (also see [Hardy and Wright 2008, Theorem 429], [Jameson 2003, Exercise 1, p. 96], and [Titchmarsh 1986,

MSC2010: primary 11N45; secondary 05C30, 05C38, 05C50.

Keywords: Ihara zeta functions, primes in graphs, Mertens' theorem. 
Equality (3.15.2)]): as $x \rightarrow \infty$,

$$
\prod_{p \leq x}\left(1-\frac{1}{p}\right) \sim \frac{e^{-\gamma}}{\log x},
$$

where $\gamma=0.57721 \ldots$ is the Euler-Mascheroni constant.

- Prime number theorem (proved by de la Vallée Poussin and Hadamard in 1896; see, e.g., [Hardy and Wright 2008, Theorem 6], [Jameson 2003, Theorem 3.4.3], and [Titchmarsh 1986, Equality (3.7.1)]): as $x \rightarrow \infty$,

$$
\pi(x) \sim \frac{x}{\log x},
$$

where $\pi(x)$ denotes the number of rational prime numbers $p$ less than $x$, that is,

$$
\pi(x):=\mid\{p: p \text { is a rational prime number with } p \leq x\} \mid .
$$

All proofs of the above formulae are related to the Riemann zeta function

$$
\zeta(s)=\prod_{p \in \mathscr{P}}\left(1-\frac{1}{p^{s}}\right)^{-1},
$$

where $\mathscr{P}$ denotes the set of all rational prime numbers, that is,

$$
\mathscr{P}:=\{p \in \mathbb{Z}: p \text { is a rational prime number }\}
$$

and to the prime zeta function, defined first by Glaisher [1891],

$$
P(s)=\sum_{p \in \mathscr{P}} \frac{1}{p^{s}} .
$$

In graph theory, there exists an analogue of the Riemann zeta function, the so-called (Ihara) zeta function $Z_{X}(u)$ of a graph $X$ (see [Ihara 1966]). Therefore, studying graph-theoretic analogues of these theorems is very interesting. Indeed, Terras and coworkers gave an analogue of the prime number theorem (see Theorem 2.10 in [Horton et al. 2006], and also Theorem 10.1 in [Terras 2011]):

If $\Delta_{X}$ divides $n$, then, as $n \rightarrow \infty$,

$$
\pi_{X}(n) \sim \frac{\Delta_{X}}{n \cdot R_{X}^{n}},
$$

and otherwise $\pi_{X}(n) \sim 0$. (For the definitions of $\pi_{X}(n)$ and $R_{X}$, see this section, and for that of $\Delta_{X}$, see Section 3.) This is called the graph-theoretic prime number theorem. 
In this paper, we define a prime zeta function of a graph, and investigate several properties of this function. In particular, we show that this has a natural boundary. Moreover, by using this function, we present graph-theoretic analogues of Mertens' theorems.

We shall note a relation between previous works and our works. A zeta function of a graph can be specialized from a dynamical zeta function for a flow (see Chapter 4 in [Terras 2011]), and dynamical-systemic analogues to the above formulae are already known (see, e.g., [Sharp 1991] for Mertens' theorems, and [Parry 1983; Parry and Pollicott 1983] for a prime number theorem). In that sense, our statements for Mertens' theorems are not new (see Remark 17). However, since our proofs are graph-theoretic and elementary, they are completely different from previous proofs.

In this section, we first recall the notation for graph theory and zeta functions of graphs, define a prime zeta function of a graph, and finally state the main theorem.

Now we recall the notation of graph theory. Throughout this paper, we always assume that $X$ is a finite, connected, non-cycle and undirected graph without degreeone vertices. Let $X$ be a graph with vertex set $V$, with $v:=|V|$, and edge set $E$, with $\epsilon:=|E|$. Simply, such a graph $X$ is denoted by $X:=(V, E)$. Note that $\epsilon$ is the number of edges of $X$.

An oriented edge (or an arc) $a$ from a vertex $u$ to a vertex $v$ is denoted by $a=(u, v)$, and the inverse of $a$ is denoted by $a^{-1}=(v, u)$. The origin and terminus of $a$ are denoted by $o(a)$ and $t(a)$, respectively. We can now orient the edges of $X$, and label the edges as follows:

$$
\vec{E}=\left\{e_{1}, e_{2}, \ldots, e_{\epsilon}, e_{\epsilon+1}=e_{1}^{-1}, e_{\epsilon+2}=e_{2}^{-1}, \ldots, e_{2 \epsilon}=e_{\epsilon}^{-1}\right\} .
$$

A path $C=a_{1} \cdots a_{s}$, where the $a_{i}$ are oriented edges, is said to have a backtrack (resp. tail) if $a_{j+1}=a_{j}^{-1}$ for some $j$ (resp. $a_{s}=a_{1}^{-1}$ ), and a path $C$ is called a cycle (or a closed path) if $o\left(a_{1}\right)=t\left(a_{s}\right)$. The length $\ell(C)$ of a path $C=a_{1} \cdots a_{s}$ is defined by $\ell(C)=s$.

A cycle $C$ is called prime (or primitive) if it satisfies the following:

- $C$ does not have backtracks or a tail;

- no cycle $D$ exists such that $C=D^{f}$ for some $f>1$.

The equivalence class $[C]$ of a cycle $C=a_{1} \cdots a_{s}$ is defined as the set of cycles

$$
[C]:=\left\{a_{1} a_{2} \cdots a_{s-1} a_{s}, a_{2} \cdots a_{s-1} a_{s} a_{1}, \ldots, a_{s} a_{1} a_{2} \cdots a_{s-1}\right\},
$$

and an equivalence class $[P]$ of a prime cycle $P$ is called a prime in the graph $X$. Throughout this paper, we denote a prime by the symbol $[P]$. Two cycles $C_{1}$ and $C_{2}$ are called equivalent if $C_{2} \in\left[C_{1}\right]$. Note that if $\left[C_{1}\right]=\left[C_{2}\right]$, then $\ell\left(C_{1}\right)=\ell\left(C_{2}\right)$, and thus $u^{\ell\left(C_{1}\right)}=u^{\ell\left(C_{2}\right)}$. 
Next, we recall the zeta function of a graph $X=\left(V=\left\{v_{1}, \ldots, v_{v}\right\}, E\right)$, and we define a prime zeta function associated with it. Let $u$ be a complex variable, and let $f_{X}(u)$ denote

$$
f_{X}(u):=\operatorname{det}\left(I_{v}-A u+Q u^{2}\right),
$$

where $I_{v}$ is the $v \times v$ identity matrix, $A$ is the adjacency matrix of $X$ (see Definition 2.1 in [Terras 2011]), and

$$
Q=\operatorname{diag}\left(\operatorname{deg}\left(v_{1}\right)-1, \ldots, \operatorname{deg}\left(v_{v}\right)-1\right) .
$$

Let $\pi_{X}(n)$ denote

$$
\pi(n)=\pi_{X}(n):=\mid\{[P]:[P] \text { is a prime in } X \text { with } \ell(P)=n\} \mid .
$$

Throughout this paper, we fix an arbitrary real number $t>1$ (that is, $\log t>0$ ), and we set $u=t^{-s}$. The (Ihara) zeta function of $X$ (see Definition 2.2 and Theorem 2.5 in [Terras 2011]) and the prime zeta function of $X$ are defined as follows:

$$
\begin{array}{lll}
Z_{X}(u):=\prod_{[P]}\left(1-u^{\ell(P)}\right)^{-1}=\frac{1}{\left(1-u^{2}\right)^{\epsilon-v} f_{X}(u)}, & \mathscr{L}_{X}(s):=Z_{X}\left(t^{-s}\right), \\
P_{X}(u):=\sum_{[P]} u^{\ell(P)}=\sum_{n=1}^{\infty} \pi_{X}(n) u^{n}, & \mathscr{P}_{X}(s):=P_{X}\left(t^{-s}\right),
\end{array}
$$

with $|u|$ sufficiently small, where $[P]$ runs through all primes in $X$. In this paper, we do not distinguish between the two functions $Z_{X}(u)$ and $\mathscr{E}_{X}(s)$, or between $P_{X}(u)$ and $\mathscr{P}_{X}(s)$. The right-hand side of the first equality is called the Ihara-Bass formula (see [Bass 1992]). Note that, owing to our assumption for $X$, the zeta function $Z_{X}(u)$ is expressible like that.

Note that, for two finite connected graphs $X_{1}$ and $X_{2}$ without degree-one vertices, $P_{X_{1}}(u)=P_{X_{2}}(u)$ if and only if $Z_{X_{1}}(u)=Z_{X_{2}}(u)$ (see Proposition 7 in [Storm 2010]).

Let

$$
T:=\bigcup_{n=1}^{\infty} T_{n} \quad \text { and } \quad T_{n}:=\left\{u \in \mathbb{C}: f_{X}\left(u^{n}\right)=0\right\}
$$

be the zeroes of the $f_{X}\left(u^{n}\right)$. Note that the elements of $T_{n}$ are poles of $Z_{X}\left(u^{n}\right)$. The radius of convergence of $Z_{X}(u)$ is denoted by $R_{X}$. Note that $0<R_{X}<1$ since $X$ is a non-cycle graph (see, e.g., [Terras 2011, p. 197]). It follows from the graph-theoretic prime number theorem (see Theorem 10.1 in [Terras 2011]) that the radius of convergence of the other function $P_{X}(u)$ is also equal to $R_{X}$. Note that the point $u=R_{X}$ is a singularity of $P_{X}(u)$, and that

$$
P_{X}(u) \sim-\log \left(R_{X}-u\right)
$$


as $u \uparrow R_{X}$, which is similar to

$$
P(s) \sim-\log (s-1)
$$

as $s \downarrow 1$ (see, e.g., [Fröberg 1968]), where $P(s)=\sum_{p} 1 / p^{s}$ denotes the prime zeta function associated with the Riemann zeta function.

Euclid proved that the number of primes $p$ is infinite. Euler showed that the prime zeta function $\sum_{p} 1 / p$ diverges, and as an application he proved the infinitude of primes. In graph theory, it is also well known that the number of primes $[P]$ in $X$ is infinite. We can give another proof "à la Euler" for this fact since $u=R_{X}$ is a singularity of $P_{X}(u)$.

Our main theorem is:

Main Theorem. Suppose that $X=(V, E)$ is a finite, connected and non-cycle graph without degree-one vertices.

(1) Let $\mu(n)$ denote the Möbius function. If $|u|<R_{X}$, then

$$
P_{X}(u)=\sum_{n=1}^{\infty} \frac{\mu(n)}{n} \log Z_{X}\left(u^{n}\right) .
$$

Furthermore, the right-hand side is absolutely convergent for $u$ satisfying $|u|<1$ and $u \notin T$, and so $P_{X}(u)$ has an analytic extension to the region $\{u \in \mathbb{C}:|u|<1\} \backslash T$.

(2) The imaginary axis $\operatorname{Re}(s)=0$ is a natural boundary for the function $\mathscr{P}_{X}(s)$, that is, every point on this line can be realized as a limit point of singularities of $\mathscr{P}_{X}(s)$.

(3) (Graph-theoretic Mertens' first theorem) As $N \rightarrow \infty$,

$$
\sum_{n \leq N} n \cdot \pi_{X}(n) R_{X}^{n}=N+O(1) .
$$

(4) (Graph-theoretic Mertens' second theorem) There exists a constant $B_{X}$ such that, as $N \rightarrow \infty$,

$$
\sum_{n \leq N} \pi_{X}(n) R_{X}^{n}=\log N+B_{X}+O\left(\frac{1}{N}\right) .
$$

(5) (Graph-theoretic Mertens' third theorem) Let $\gamma=0.57721 \ldots$ denote the Euler-Mascheroni constant. As $N \rightarrow \infty$,

$$
\prod_{\ell(P) \leq N}\left(1-R_{X}^{\ell(P)}\right) \sim \frac{e^{-\gamma}}{C_{X}} \cdot \frac{1}{N},
$$


where

$$
C_{X}=-\frac{1}{\left(1-R_{X}^{2}\right)^{\epsilon-v} R_{X} f_{X}^{\prime}\left(R_{X}\right)}
$$

(for the definition, in detail, see Section 3 in this paper).

The contents of this paper are as follows. In the next section, we prove the first two claims in the main theorem, that is, several properties of $P_{X}(u)$. In Section 3, we prove the remaining claims in the main theorem, namely, the graph-theoretic Mertens theorems.

\section{Prime zeta function of a graph}

In this section, we give a proof of parts (1) and (2) of the Main Theorem introduced in Section 1.

The following facts about $Z_{X}(u)$, etc., are known, and are often used in this paper.

Facts 1. (1) (Basic facts) For an arbitrary real number $t>1$, set $u=t^{-s}$. Then the function $\mathscr{L}_{X}(s)$ is absolutely convergent and holomorphic for all s satisfying $\operatorname{Re}(s)>-\log R_{X} / \log t(\geq 0)$.

Since the function $Z_{X}(u)$ is the reciprocal of a polynomial by the Ihara-Bass formula, the function $Z_{X}(u)$ is meromorphic for all $u \in \mathbb{C}$, and therefore $\mathscr{L}_{X}(s)$ is also meromorphic for all $s \in \mathbb{C}$.

(2) [Kotani and Sunada 2000, Theorem 1.3(1)] Let $q+1$ and $p+1$ be the maximum and minimum degrees of a graph $X$, respectively. Then $1 / q \leq R_{X} \leq 1 / p$, the point $u=R_{X}$ is a simple pole of $Z_{X}(u)$, and every pole of $Z_{X}(u)$ satisfies $R_{X} \leq|u| \leq 1$.

(3) [Terras 2011, p. 197] Suppose that $X$ is a finite connected graph without degreeone vertices. Then $R_{X}=1$ if and only if $X$ is a cycle graph. This follows from the equation $p=q=1$.

(4) [Kotani and Sunada 2000, p. 8] The leading coefficient of the polynomial $f_{X}$ is given by

$$
c=\prod_{v \in V}(\operatorname{deg}(v)-1),
$$

and therefore that of the polynomial $1 / Z_{X}$ is equal to $c_{2 \epsilon}=(-1)^{\epsilon-v} c$.

In this section, the following lemma is important.

Key Lemma 2. Let

$$
\phi(u)=1+\sum_{i=1}^{d} c_{i} u^{i} \in \mathbb{Z}[u]
$$


be a polynomial function of degree $d \geq 0$, and let

$$
T=\left\{u \in \mathbb{C}: \text { there exists } n \geq 1 \text { such that } \phi\left(u^{n}\right)=0\right\}
$$

denote the zeroes of the $\phi\left(u^{n}\right)$. Suppose that $r$ is an arbitrary real number, and assume that $\Phi(u)$ is a series defined by

$$
\Phi(u)=\sum_{n=1}^{\infty} \frac{1}{n^{r}} \log \phi\left(u^{n}\right) .
$$

Then $\Phi(u)$ is absolutely convergent for $u$ satisfying $|u|<1$ and $u \notin T$.

Proof. First, we suppose that $d=0$. Then the $\phi\left(u^{n}\right)=1$ are constant, and therefore $\Phi(u)=0$ is also constant. Hence, the claim is trivial. From now on, we assume that $d \geq 1$. Set $c:=\max \left\{\left|c_{i}\right|: 1 \leq i \leq d\right\}$, choose a number $C_{0}$ with $C_{0} \geq c d+1$ $(\geq 2)$, and fix it.

Let $r_{n}(n \geq 3)$ be a number defined by

$$
r_{n}:=\left(\frac{1-\exp \left(-1 / n^{2-r}\right)}{C_{0}}\right)^{1 / n} .
$$

Note that $r_{n}<\left(1 / C_{0}\right)^{1 / n}$, the sequence $\left\{r_{n}\right\}_{n \geq 3}$ is increasing, and $\lim _{n \rightarrow \infty} r_{n}=1$.

Take $u$ satisfying $|u|<1$ and $u \notin T$, and fix it. Then there exists a number $N$ such that $|u| \leq r_{N}$, and thus $|u|<r_{n}$ for all $n \geq N+1$. Now we fix such numbers $N$ and $n$.

Since $|u|<\left(1 / C_{0}\right)^{1 / n}$ and $\left|u^{n}\right| \leq|u|<1$, we obtain, by the triangle inequality,

$$
0<1-C_{0}\left|u^{n}\right| \leq\left|\phi\left(u^{n}\right)\right|, \quad \text { and so } \quad-\log \left|\phi\left(u^{n}\right)\right| \leq-\log \left(1-C_{0}\left|u^{n}\right|\right) .
$$

On the other hand, since $|u|<r_{n}$, then $C_{0}\left|u^{n}\right|<1-\exp \left(-1 / n^{2-r}\right)$, so we obtain the inequality $-\log \left(1-C_{0}\left|u^{n}\right|\right)<1 / n^{2-r}$. Combining this result with (1), we obtain

$$
\operatorname{Re}\left(-\log \phi\left(u^{n}\right)\right)=-\log \left|\phi\left(u^{n}\right)\right|<\frac{1}{n^{2-r}} .
$$

The first inequality in (1) also shows that the function $\log \phi\left(u^{n}\right)$ is holomorphic in the closed disk $|u| \leq r_{N+1}$. By applying the Borel-Carathéodory theorem (see, e.g., [Titchmarsh 1958, §5.5]) to the function $\log \phi\left(u^{n}\right)$ and the two circles $|u|=r_{N+1}$, $|u|=r_{N}$, we obtain

$$
\left|\log \phi\left(u^{n}\right)\right| \leq \max _{|u|=r_{N}}\left|\log \phi\left(u^{n}\right)\right| \leq K \max _{|u|=r_{N+1}} \operatorname{Re}\left(-\log \phi\left(u^{n}\right)\right) \leq K \frac{1}{n^{2-r}},
$$

where $K:=2 r_{N} /\left(r_{N+1}-r_{N}\right)$. Therefore, it follows that

$$
\sum_{n=N+1}^{\infty} \frac{1}{n^{r}}\left|\log \phi\left(u^{n}\right)\right| \leq K \sum_{n=N+1}^{\infty} \frac{1}{n^{2}}<K \cdot \zeta(2)<\infty .
$$


Hence, for $u$ satisfying $|u|<1$ and $u \notin T$, the series $\Phi(u)$ converges absolutely.

Using this lemma, we can prove the following proposition.

Proposition 3. Let $\mu(n)$ denote the Möbius function. If $|u|<R_{X}$, then

$$
P_{X}(u)=\sum_{n=1}^{\infty} \frac{\mu(n)}{n} \log Z_{X}\left(u^{n}\right) .
$$

Moreover, the right-hand side of (3) is absolutely convergent for $u$ satisfying $|u|<1$ and $u \notin T$, and therefore $P_{X}(u)$ extends analytically to the region $\{u \in \mathbb{C}:|u|<1\} \backslash T$.

Equivalently, if $\operatorname{Re}(s)>-\log R_{X} / \log t$, then

$$
\mathscr{P}_{X}(s)=\sum_{n=1}^{\infty} \frac{\mu(n)}{n} \log \mathscr{L}_{X}(n s) .
$$

The right-hand side of (4) is absolutely convergent for $s$ satisfying $\operatorname{Re}(s)>0$ and $t^{-s} \notin T$, and so (4) gives the analytic continuation of $\mathscr{P}_{X}(s)$ to the region.

Proof. Note that $R_{X} \leq 1$ (from Fact 1(2)) and $\exp (z)=\prod_{n=1}^{\infty}\left(1-z^{n}\right)^{-\mu(n) / n}$ for $|z|<1$. Suppose that $|u|<R_{X}$. Since $\left|u^{\ell(P)}\right| \leq|u|<1$, we obtain the equality

$$
\exp \left(P_{X}(u)\right)=\prod_{[P]} \exp \left(u^{\ell(P)}\right)=\prod_{[P]} \prod_{n=1}^{\infty}\left(1-u^{n \ell(P)}\right)^{-\mu(n) / n}=\prod_{n=1}^{\infty} Z_{X}\left(u^{n}\right)^{\mu(n) / n},
$$

and therefore (3) holds for $u$ satisfying $|u|<R_{X}$.

Set

$$
1 / Z_{X}(u)=\left(1-u^{2}\right)^{\epsilon-v} f_{X}(u)=1+c_{1} u+\cdots+c_{2 \epsilon} u^{2 \epsilon} \in \mathbb{Z}[x],
$$

$c=\max \left\{\left|c_{i}\right|: 1 \leq i \leq 2 \epsilon\right\}$ and $C_{0}=2 \epsilon c \geq 2$. By applying Key Lemma 2 to $\phi(u)=1 / Z_{X}(u)$ and $r=1$, it follows that, for $u$ satisfying $|u|<1$ and $u \notin T$, the series $\sum_{n=1}^{\infty} \log Z_{X}\left(u^{n}\right) / n$ is absolutely convergent, and so the right-hand side of (3) is absolutely convergent.

Moreover, for a Ramanujan graph, we can prove the following.

Corollary 4. Suppose that $X$ is a finite connected Ramanujan graph with degree $q+1$, that is, $Z_{X}(u)$ satisfies the Riemann hypothesis (see Theorem 7.4 in [Terras 2011]). Then the function $P_{X}(u)$ is absolutely convergent for $u$ satisfying $|u|<1$ and $|u| \neq(1 / q)^{1 / n}$ for all $n$.

Equivalently, the function $\mathscr{P}_{X}(s)$ is absolutely convergent for $s$ such that $\operatorname{Re}(s)>0$ and $\operatorname{Re}(s) \neq \log q / \log t^{n}$ for all $n$.

Proof. Since $X$ is a Ramanujan graph, by Theorem 1.3 in [Kotani and Sunada 2000], every real (resp. nonreal) zero of $f_{X}(u)$ satisfies $|u|=1$ or $1 / q($ resp. $|u|=1 / \sqrt{q})$. Thus, every point $|u| \neq(1 / q)^{1 / n}$ is not zero of $f_{X}\left(u^{n}\right)$. Hence, the proof of the assertion follows from Proposition 3. 
We can completely interchange the roles of the functions $P_{X}(u)$ and $\log Z_{X}(u)$.

Corollary 5. If $|u|<1$ and $u \notin T$, then

$$
\log Z_{X}(u)=\sum_{n=1}^{\infty} \frac{1}{n} P_{X}\left(u^{n}\right) .
$$

Equivalently, if $\operatorname{Re}(s)>0$ and $t^{-s} \notin T$, then

$$
\log \mathscr{L}_{X}(s)=\sum_{n=1}^{\infty} \frac{1}{n} \mathscr{P}_{X}(n s) .
$$

Proof. By applying the Möbius inversion formula (see, e.g., Theorem 270 in [Hardy and Wright 2008], or Theorem 2.2.8 in [Jameson 2003]) to the equality (3) for $|u|<1$, we obtain the equality (5).

Remark 6. The equalities (4) and (6) indicate that $\mathscr{P}_{X}(s)$ is a graph-theoretic analogue to the prime zeta function $P(s)$ for the Riemann zeta function $\zeta(s)$. The relations between $P(s)$ and $\zeta(s)$ are given as follows (see [Glaisher 1891], and also [Fröberg 1968] and Equality (1.6.1) in [Titchmarsh 1986]):

For $\operatorname{Re}(s)>1$,

$$
P(s)=\sum_{n=1}^{\infty} \frac{\mu(n)}{n} \log \zeta(n s) \quad \text { and } \quad \log \zeta(s)=\sum_{n=1}^{\infty} \frac{1}{n} P(n s) .
$$

We can orient the edges of $X$, and label the edges as follows:

$$
\vec{E}=\left\{a_{1}, a_{2}, \ldots, a_{\epsilon}, a_{\epsilon+1}=a_{1}^{-1}, a_{\epsilon+2}=a_{2}^{-1}, \ldots, a_{2 \epsilon}=a_{\epsilon}^{-1}\right\} .
$$

Let $W=W_{X}:=\left(w_{i j}\right)$ denote the edge adjacency matrix of a graph $X$, that is, a $2 \epsilon \times 2 \epsilon$ matrix defined by

$$
w_{i j}:= \begin{cases}1 & \text { if } t\left(a_{i}\right)=o\left(a_{j}\right) \text { and } a_{j} \neq a_{i}^{-1} \text { for } a_{i}, a_{j} \in \vec{E}, \\ 0 & \text { otherwise }\end{cases}
$$

(see p. 28 in [Terras 2011]). Let $\lambda_{1}, \ldots, \lambda_{k}$ be the distinct eigenvalues of $W$, and let $e_{1}, \ldots, e_{k}$ be their multiplicities. Note that $\sum_{i=1}^{k} e_{i}=2 \epsilon$. Let $e:=\sum_{i=1, \lambda_{i} \neq \pm 1}^{k} e_{i}$. By the determinant formula given by Hashimoto [1989] and Bass [1992], the polynomial $1 / Z_{X}(u)$ can be written as

$$
1 / Z_{X}(u)=\operatorname{det}\left(I_{2 \epsilon}-W u\right)=\prod_{i=1}^{k}\left(1-\lambda_{i} u\right)^{e_{i}} .
$$

Note that $f_{X}(1)=0$. We now define a polynomial $g_{X}(u)$ by

$$
g_{X}(u):=f_{X}(u) /(1-u) .
$$


Note that since $f_{X}^{\prime}(1)=2(\epsilon-v) \kappa$ by [Northshield 1998, Theorem],

$$
g_{X}(1)=-f_{X}^{\prime}(1)=-2(\epsilon-v) \kappa,
$$

where $\kappa$ is the complexity of $X$, that is, the number of spanning trees in $X$. Since $X$ is a non-cycle graph, that is, $\epsilon \neq \nu$, the polynomial $g_{X}(u)$ can be also written as

$$
g_{X}(u)=\frac{1 / Z_{X}(u)}{\left(1-u^{2}\right)^{\epsilon-v}(1-u)}=(1+u)^{2 v-1-e} \prod_{\substack{i=1 \\ \lambda_{i} \neq \pm 1}}^{k}\left(1-\lambda_{i} u\right)^{e_{i}} .
$$

We can show that the function $\mathscr{P}_{X}(s)$ has a natural boundary.

Proposition 7. Let $X=(V, E)$ be a finite, connected and non-cycle graph without degree-one vertices.

(1) There exists an eigenvalue $\lambda$ of $W$ such that $|\lambda|>1$.

(2) The imaginary axis $\operatorname{Re}(s)=0$ is a natural boundary for the function $\mathscr{P}_{X}(s)$, that is, every point on this line can be realized as a limit point of singularities of $\mathscr{P}_{X}(s)$.

Proof. (1) The leading coefficient $c_{2 \epsilon}$ of the polynomial $1 / Z_{X}(u)$ is given by

$$
(-1)^{\epsilon-v} \prod_{v \in V}(\operatorname{deg}(v)-1)=c_{2 \epsilon}=\prod_{i=1}^{k} \lambda_{i}^{e_{i}}
$$

(from Fact 1(4)). By our assumption for $X$, the graph $X$ is not a 2-regular graph. Thus $\left|c_{2 \epsilon}\right|>1$ and so there exists an eigenvalue $\lambda_{i}$ with $\left|\lambda_{i}\right| \neq 1$. Note that every pole $1 / \lambda_{i}$ of $Z_{X}(u)$ satisfies $\left|1 / \lambda_{i}\right| \leq 1$ by Fact $1(2)$. So there exists an eigenvalue $\lambda_{i}$ with $\left|\lambda_{i}\right|>1$.

(2) Note that $\exp (z)=\prod_{n=1}^{\infty}\left(1-z^{n}\right)^{-\mu(n) / n}$ for $|z|<1$. If $|u|<1$ and $u \notin T$, then

$$
\begin{aligned}
\exp \left(P_{X}(u)\right) & =\prod_{n=1}^{\infty} Z_{X}\left(u^{n}\right)^{\mu(n) / n} \\
& =\left(\prod_{n=1}^{\infty}\left(1-u^{2 n}\right)^{-\mu(n) / n}\right)^{\epsilon-v}\left(\prod_{n=1}^{\infty}\left(1-u^{n}\right)^{-\mu(n) / n}\right) \prod_{n=1}^{\infty} g_{X}\left(u^{n}\right)^{-\mu(n) / n} \\
& =\exp \left((\epsilon-v) u^{2}+u\right) \prod_{n=1}^{\infty} g_{X}\left(u^{n}\right)^{-\mu(n) / n}
\end{aligned}
$$

and therefore the equality

$$
P_{X}(u)=(\epsilon-v) u^{2}+u-\sum_{n=1}^{\infty} \frac{\mu(n)}{n} \log g_{X}\left(u^{n}\right)
$$

holds. 
Note that $u=t^{-s}$. By using the equalities (7) and 2, the function $\mathscr{P}_{X}(s)$ can be written as

$$
\begin{aligned}
\mathscr{P}_{X}(s)=(\epsilon-v) t^{-2 s}+t^{-s} & \\
& -\sum_{n=1}^{\infty} \frac{\mu(n)}{n}\left((2 v-1-e) \log \left(1+t^{-n s}\right)+\sum_{\substack{i=1 \\
\lambda_{i} \neq \pm 1}}^{k} e_{i} \log \left(1-\lambda_{i} t^{-n s}\right)\right)
\end{aligned}
$$

for all $s$ satisfying $\operatorname{Re}(s)>0$. By part (1), there exists $\lambda$ such that $|\lambda|>1$ among the eigenvalues $\lambda_{1}, \ldots, \lambda_{k}$ of $W$. Note that $1-\lambda t^{-n s}=0$ if and only if $s=r(\lambda, n, m)$, where

$$
r(\lambda, n, m):=\frac{\log |\lambda|}{n \log t}+i \frac{\operatorname{Arg}(\lambda)+2 \pi m}{n \log t},
$$

and $\operatorname{Arg}(\lambda)$ is the argument of $\lambda$ with $-\pi \leq \operatorname{Arg}(\lambda)<\pi$. Note that

$$
\varepsilon_{n}:=\frac{\log |\lambda|}{n \log t} \rightarrow 0
$$

as $n \rightarrow \infty$. We now fix an arbitrary point $\alpha=i a$ on the imaginary axis $\operatorname{Re}(s)=0$. Then, we can arrange a sequence of integers $\left\{m_{n}\right\}$ for each integer $n$ so that

$$
\frac{\operatorname{Arg}(\lambda)+2 \pi m_{n}}{n \log t} \rightarrow a
$$

as $n \rightarrow \infty$. Hence, each point $\alpha$ on the boundary is a limit point of singularities of $\mathscr{P}_{X}(s)$. Since $\varepsilon_{n}>0$ for all $n$, we cannot continue $\mathscr{P}_{X}(s)$ beyond the boundary at $\operatorname{Re}(s)=0$.

Remark 8. Proposition 7(2) is an analogue of the fact that the imaginary axis $\operatorname{Re}(s)=0$ is a natural boundary for the prime zeta function $P(s)$ of the Riemann zeta function $\zeta(s)$ (see [Landau and Walfisz 1920]).

\section{Graph-theoretic Mertens' theorem}

In this section, we prove parts (3)-(5) of the Main Theorem introduced in Section 1.

Throughout this section, we always assume that $X=(V, E)$ is a finite, connected, non-cycle graph without degree-one vertices. Note in particular that $v \neq \epsilon$ and $0<R_{X}<1$.

First, we define the constants $H_{X}, C_{X}$ and $\gamma_{X}$, and study their properties, which play important roles in this section. Let $u$ be a complex variable. We define a function by

$$
H_{X}(u):=\log Z_{X}(u)-P_{X}(u)=\sum_{n \geq 2} \frac{1}{n} P_{X}\left(u^{n}\right)=\sum_{[P]} \sum_{n \geq 2} \frac{1}{n} u^{n \ell(P)} .
$$


Note that the point $u=R_{X}$ is a common pole of $Z_{X}(u)$ and $P_{X}(u)$ by Fact 1(2), and that the series $H_{X}(u)$ is absolutely convergent for $u$ satisfying $|u|<1$ and $u \notin T$, from Corollary 5.

Since $u=R_{X}$ is a simple pole of $Z_{X}(u)$, we can define constants $c_{X}$ and $C_{X}$ by

$$
c_{X}:=-\operatorname{Res}_{u=R_{X}} Z_{X}(u)=\lim _{u \uparrow R_{X}}\left(R_{X}-u\right) Z_{X}(u)=\frac{-1}{\left(1-R_{X}^{2}\right)^{\epsilon-v} f_{X}^{\prime}\left(R_{X}\right)}
$$

and $C_{X}:=c_{X} / R_{X}$.

Lemma 9. (1) The value $H_{X}:=H_{X}\left(R_{X}\right)$ is finite.

(2) The constants $c_{X}$ and $C_{X}$ are positive.

Proof. (1) Since $R_{X}^{n}<R_{X}<1(n \geq 2)$, the function $P_{X}(u)$ is holomorphic at $u=R_{X}^{n}$, and therefore $P_{X}\left(u^{n}\right)$ is holomorphic at $u=R_{X}$. We have

$$
\begin{aligned}
H_{X}\left(R_{X}\right) & =\sum_{[P]} \sum_{n \geq 2} \frac{1}{n} R_{X}^{n \ell(P)} \leq \sum_{[P]} \sum_{n \geq 2} R_{X}^{n \ell(P)} \\
& =\sum_{[P]} \frac{R_{X}^{2 \ell(P)}}{1-R_{X}^{\ell(P)}} \leq \frac{1}{1-R_{X}} \sum_{[P]} R_{X}^{2 \ell(P)}=\frac{P_{X}\left(R_{X}^{2}\right)}{1-R_{X}}<+\infty,
\end{aligned}
$$

and the assertion follows.

(2) Note that the leading coefficient of the polynomial $f_{X}$ is given by

$$
c=\prod_{v \in V}(\operatorname{deg}(v)-1)>0
$$

by Fact $1(4)$. Then $f_{X}$ factors as the product of irreducible polynomials such that

$$
f_{X}(u)=c \prod_{i=1}^{m_{1}}\left(u-\alpha_{i}\right) \cdot \prod_{j=1}^{m_{2}} f_{j}(u),
$$

where the $f_{j}$ are monic of $\operatorname{deg} f_{j}=2$, and $\operatorname{deg} f_{X}=2 v=m_{1}+2 m_{2}$. Note that $m_{1}$ is even. Since $u=R_{X}$ is a simple pole of $Z_{X}(u)$, it is a simple zero of $f_{X}$. We may assume that $\alpha_{1}=R_{X}$. Since $\alpha_{i}>R_{X}\left(2 \leq i \leq m_{1}\right)$ and the discriminants of the $f_{j}$ are negative, the sign of

$$
f_{X}^{\prime}\left(R_{X}\right)=c \prod_{i=2}^{m_{1}}\left(R_{X}-\alpha_{i}\right) \prod_{j=1}^{m_{2}} f_{j}\left(R_{X}\right)
$$

is equal to $(-1)^{m_{1}-1}=-1$, i.e., $f_{X}^{\prime}\left(R_{X}\right)<0$, so $c_{X}>0$ and $C_{X}=c_{X} / R_{X}>0$. 
Since the function $Z_{X}(u)-c_{X} /\left(R_{X}-u\right)$ is holomorphic at $u=R_{X}$, we can define a constant $\gamma_{X}$ by

$$
\gamma_{X}:=\lim _{u \uparrow R_{X}}\left(Z_{X}(u)-\frac{c_{X}}{R_{X}-u}\right),
$$

which is an analogue of the Euler-Mascheroni constant $\gamma=\lim _{s \downarrow 1}(\zeta(s)-1 /(s-1))$ for $\zeta(s)$.

In a neighborhood of $u=R_{X}$, the function $Z_{X}(u)$ can be expanded as

$$
Z_{X}(u)=\frac{c_{X}}{R_{X}-u}+\gamma_{X}+O\left(R_{X}-u\right),
$$

and so

$$
\log Z_{X}(u)=\log \frac{c_{X}}{R_{X}-u}+O\left(R_{X}-u\right) .
$$

Similarly, in a neighborhood of $u=R_{X}$, the function $P_{X}(u)$ can be expanded as $P_{X}(u)=\log \frac{c_{X}}{R_{X}-u}-H_{X}(u)+O\left(R_{X}-u\right)=\log \frac{c_{X}}{R_{X}-u}-H_{X}\left(R_{X}\right)+O\left(R_{X}-u\right)$.

In this section, the following facts are used.

Facts 10. (1) (See, for example, Theorem 18.1 in [Korevaar 2002].) Let $x$ be a complex variable and let $F(x)=\sum_{n=0}^{\infty} a_{n} x^{n}$ be a power series with $a_{n} \geq 0$ that converges for $|x|<1$. Suppose that

$$
F(x)-\frac{C}{1-x}=O(1)
$$

as $x \rightarrow 1$. Then the partial sum $A(N)=\sum_{n \leq N} a_{n}$ satisfies

$$
A(N)=C \cdot N+O(\log N)
$$

as $N \rightarrow \infty$.

(2) (See, for example, Exercises 9-6 in [Apostol 1974], and Theorem 1.3.6 in [Jameson 2003], the Abel partial summation formula). Let $\left\{a_{n}\right\}$ be real numbers, and let $f(t)$ be a (real-or complex-valued) function with a continuous derivative in the interval $[1, N]$. Then

$$
\sum_{n \leq N} a_{n} f(n)=A(N) f(N)-\int_{1}^{N} A(t) f^{\prime}(t) d t .
$$

By using Fact 10, we can prove the following proposition.

Proposition 11. Suppose that $X$ is a finite, connected and non-cycle graph without degree-one vertices. In a neighborhood of $u=R_{X}$, expand $Z_{X}(u)$ into the 
power series

$$
Z_{X}(u)=\sum_{n=0}^{\infty} a_{n}^{\prime} u^{n}
$$

Then, as $N \rightarrow \infty$,

$$
\sum_{n \leq N} a_{n}^{\prime} R_{X}^{n}=C_{X} \cdot N+O(\log N)
$$

Proof. First, for simplicity of arguments, we normalize the function $Z_{X}(u)$ :

$$
F(x)=Z_{X}\left(R_{X} x\right)=\sum_{n=0}^{\infty} a_{n}^{\prime} R_{X}^{n} x^{n}=\sum_{n=0}^{\infty} a_{n} x^{n},
$$

where $a_{n}=a_{n}^{\prime} R_{X}^{n}$. Note that the normalized function $F(x)$ converges for $|x|<1$. Since all coefficients $a_{n}^{\prime}$ are nonnegative (by page 13 in [Terras 2011]), all coefficients $a_{n}$ are also nonnegative. Since $X$ is a non-cycle graph, the point $x=1$ is a simple pole of $F(x)$. Hence, we obtain

$$
F(x)-\frac{C_{X}}{1-x}=O(1)
$$

as $x \rightarrow 1$. By applying Fact 10(1) to this equality, as $N \rightarrow \infty$,

$$
\sum_{n \leq N} a_{n}=C_{X} \cdot N+O(\log N), \quad \text { and so } \quad \sum_{n \leq N} a_{n}^{\prime} R_{X}^{n}=C_{X} \cdot N+O(\log N)
$$

holds, and the assertion follows.

Now, we compute the following example.

Example 12 [Terras 2011, Example 2.8, p. 18]. Consider the graph $X=K_{4}-$ \{one edge\}. Then

$f_{X}(u)=(1-u)\left(1+u^{2}\right)\left(1+u+2 u^{2}\right)\left(1-u^{2}-2 u^{3}\right) \quad$ and $\quad Z_{X}(u)^{-1}=\left(1-u^{2}\right) f_{X}(u)$.

Since the radius of convergence $R_{X}$ of $Z_{X}(u)$ is the smallest positive real zero of $f_{X}(u)$,

$$
R_{X}=\frac{1}{6}\left(\alpha-1+\alpha^{-1}\right)=0.6572981 \ldots, \quad \alpha=(53+6 \sqrt{78})^{1 / 3} .
$$

Then $C_{X}$ is computed as $C_{X}=0.5540954 \ldots$ For example, if $N=50000$, then

$$
\frac{1}{N} \sum_{n \leq N} a_{n}^{\prime} R_{X}^{n}=0.5540867 \ldots \approx C_{X} .
$$


Let $X=(V, E)$ be a graph, and set $|V|=v$ and $|E|=\epsilon$. Let $W=W_{X}$ be the edge adjacency matrix of $X$ (see page 28 in [Terras 2011], or Section 2 in this paper), and let $\operatorname{Spec}(W)$ denote the spectrum of $W$, that is, the list of its eigenvalues together with their multiplicities. Note that $|\operatorname{Spec}(W)|=2 \epsilon$. The polynomial $1 / Z_{X}(u)$ has an expression different from that in Section 2. In fact, this can be written as

$$
1 / Z_{X}(u)=\operatorname{det}\left(I_{2 \epsilon}-W u\right)=\prod_{\lambda \in \operatorname{Spec}(W)}(1-\lambda u) \quad\left(=\prod_{i=1}^{k}\left(1-\lambda_{i} u\right)^{e_{i}}\right) .
$$

Since the points $u=1 / \lambda$ are the poles of $Z_{X}(u)$, we obtain $1 \leq|\lambda| \leq 1 / R_{X}$ by Fact 1(2).

The following lemma is used in the proof of Theorem 14 in this section.

Key Lemma 13. Suppose that $X$ is a finite, connected and non-cycle graph without degree-one vertices.

(1) As $N \rightarrow \infty$, we have

$$
\sum_{n=1}^{N} \sum_{\lambda \in \operatorname{Spec}(W)}\left(\lambda R_{X}\right)^{n}=N+O(1)
$$

(2) Let $0<\alpha<\frac{1}{2}$ be a fixed real number. Then there exists a natural number $N_{0}$ such that, for any $n \geq N_{0}$,

$$
\left|n \cdot \pi(n)-\sum_{\lambda \in \operatorname{Spec}(W)} \lambda^{n}\right|<2 \epsilon\left(\frac{1}{R_{X}}\right)^{(1-\alpha) n} .
$$

Proof. (1) Let $\Delta_{X}$ denote

$$
\Delta=\Delta_{X}:=\operatorname{gcd}\{\ell(P):[P] \text { is a prime in } X\}
$$

(see Definition 2.12 in [Terras 2011]). It follows from Theorem 1.4 in [Kotani and Sunada 2000] that the poles of $Z_{X}(u)$ on the circle $|u|=R_{X}$ have the form $u=R_{X} e^{2 \pi i a / \Delta}(1 \leq a \leq \Delta)$. It is well known that

$$
\sum_{a=1}^{\Delta} e^{2 \pi i a n / \Delta}= \begin{cases}\Delta & \text { if } \Delta \mid n, \\ 0 & \text { otherwise }\end{cases}
$$

(see, e.g., Exercise 10.1 in [Terras 2011]). Then we obtain

$$
\left|N-\sum_{|\lambda|=1 / R_{X}} \sum_{n=1}^{N}\left(\lambda R_{X}\right)^{n}\right|=\left|N-\sum_{n=1}^{N} \sum_{a=1}^{\Delta} e^{2 \pi i a n / \Delta}\right|=N-\left[\frac{N}{\Delta}\right] \Delta<\Delta,
$$


where $[r]$ denotes the integer part of the real number $r$. On the other hand, we obtain

$$
\left|\sum_{|\lambda|<1 / R_{X}} \sum_{n=1}^{N}\left(\lambda R_{X}\right)^{n}\right|<2 \epsilon \sum_{n \geq 1}\left(\rho R_{X}\right)^{n}=\frac{2 \epsilon \rho R_{X}}{1-\rho R_{X}},
$$

where

$$
\rho:=\max \left\{|\lambda|: \lambda \in \operatorname{Spec}(W),|\lambda|<1 / R_{X}\right\} .
$$

Combining these inequalities, by the triangle inequality we obtain

$$
\left|N-\sum_{n=1}^{N} \sum_{\lambda \in \operatorname{Spec}(W)}\left(\lambda R_{X}\right)^{n}\right|<\Delta+\frac{2 \epsilon \rho R_{X}}{1-\rho R_{X}}
$$

as $N \rightarrow \infty$, and the assertion follows.

(2) Let $\mu(n)$ denote the Möbius function. Note that $\sum_{d \mid n}|\mu(d)| \leq n$. It is known that

$$
\pi(n)=\frac{1}{n} \sum_{d \mid n} \mu(d) N_{n / d} \quad \text { and } \quad N_{n}=\sum_{\lambda \in \operatorname{Spec}(W)} \lambda^{n}
$$

(see (10.3) and (10.4) in [Terras 2011]). Combining these equalities, we obtain

$$
n \cdot \pi(n)=\sum_{\lambda \in \operatorname{Spec}(W)} \sum_{d \mid n} \mu(d) \lambda^{n / d},
$$

and thus

$$
\begin{aligned}
\left|n \cdot \pi(n)-\sum_{\lambda \in \operatorname{Spec}(W)} \lambda^{n}\right| & =\left|\sum_{\lambda \in \operatorname{Spec}(W)} \sum_{\substack{d \mid n \\
d \geq 2}} \mu(d) \lambda^{n / d}\right| \\
& \leq \sum_{\lambda \in \operatorname{Spec}(W)} \sum_{\substack{d \mid n \\
d \geq 2}}|\mu(d)| \cdot|\lambda|^{n / d} \leq \sum_{\lambda \in \operatorname{Spec}(W)} \sum_{\substack{d \mid n \\
d \geq 2}}|\mu(d)| \cdot|\lambda|^{n / 2} \\
& \leq n \sum_{\lambda \in \operatorname{Spec}(W)}\left(\frac{1}{R_{X}}\right)^{n / 2} \leq 2 \epsilon n\left(\frac{1}{R_{X}}\right)^{n / 2} .
\end{aligned}
$$

On the other hand, since $R_{X}<1$ and $0<\alpha<\frac{1}{2}$ by our assumptions, there exists a natural number $N_{0}$ such that, for any $n \geq N_{0}$,

$$
n \leq\left(\frac{1}{R_{X}}\right)^{(1 / 2-\alpha) n}, \quad \text { and so } n\left(\frac{1}{R_{X}}\right)^{n / 2} \leq\left(\frac{1}{R_{X}}\right)^{(1-\alpha) n} .
$$

Hence, for any $n \geq N_{0}$,

$$
\left|n \cdot \pi(n)-\sum_{\lambda \in \operatorname{Spec}(W)} \lambda^{n}\right| \leq 2 \epsilon\left(\frac{1}{R_{X}}\right)^{(1-\alpha) n},
$$


and the assertion follows.

At last, we can prove the main theorem in this section.

Theorem 14. Suppose that $X$ is a finite, connected and non-cycle graph without degree-one vertices. Let $\gamma=0.57721 \ldots$ be the Euler-Mascheroni constant, and let $H_{X}=H_{X}\left(R_{X}\right)$ and $C_{X}$ be the constants.

(1) (Graph-theoretic Mertens' first theorem) As $N \rightarrow \infty$,

$$
\sum_{n \leq N} n \cdot \pi(n) R_{X}^{n}=N+O(1) .
$$

(2) (Graph-theoretic Mertens' second theorem) There exists a constant $B_{X}$ such that, as $N \rightarrow \infty$,

$$
\sum_{n \leq N} \pi(n) R_{X}^{n}=\log N+B_{X}+O\left(\frac{1}{N}\right) .
$$

(3) The equality $B_{X}=\gamma+\log C_{X}-H_{X}$ holds. Equivalently,

$$
\begin{aligned}
B_{X} & =\gamma+\log C_{X}-\sum_{[P]} \sum_{n \geq 2} \frac{1}{n} R_{X}^{n \ell(P)} \\
& =\gamma+\log C_{X}+\prod_{[P]}\left(\log \left(1-R_{X}^{\ell(P)}\right)+R_{X}^{\ell(P)}\right) .
\end{aligned}
$$

(4) (Graph-theoretic Mertens' third theorem) As $N \rightarrow \infty$,

$$
\prod_{\ell(P) \leq N}\left(1-R_{X}^{\ell(P)}\right)=\prod_{n \leq N}\left(1-R_{X}^{n}\right)^{\pi(n)} \sim \frac{e^{-\gamma}}{C_{X}} \cdot \frac{1}{N} .
$$

Proof. (1) Let $N_{0}$ be a number as in the proof of Key Lemma 13(2), and let $K$ denote the constant

$$
K:=\left|\sum_{n=1}^{N_{0}-1} n \cdot \pi(n) R_{X}^{n}-\sum_{n=1}^{N_{0}-1} \sum_{\lambda \in \operatorname{Spec}(W)}\left(\lambda R_{X}\right)^{n}\right| .
$$

Assume that $N$ is sufficiently large. Then it follows from Key Lemma 13(2) that

$$
\begin{aligned}
\left|\sum_{n=1}^{N} n \cdot \pi(n) R_{X}^{n}-\sum_{n=1}^{N} \sum_{\lambda \in \operatorname{Spec}(W)}\left(\lambda R_{X}\right)^{n}\right| & \leq K+\left|\sum_{n=N_{0}}^{N} R_{X}^{n}\left(n \cdot \pi(n)-\sum_{\lambda \in \operatorname{Spec}(W)} \lambda^{n}\right)\right| \\
& \leq K+2 \epsilon \sum_{n=N_{0}}^{N} R_{X}^{\alpha n}<K+\frac{2 \epsilon}{1-R_{X}^{\alpha}},
\end{aligned}
$$


and therefore by Key Lemma 13(1) we have

$$
\sum_{n=1}^{N} n \cdot \pi(n) R_{X}^{n}=\sum_{n=1}^{N} \sum_{\lambda \in \operatorname{Spec}(W)}\left(\lambda R_{X}\right)^{n}+O(1)=N+O(1) \quad \text { as } N \rightarrow \infty
$$

(2) We set $a_{n}=n \cdot \pi(n) R_{X}^{n}$. By part (1), we obtain $A(t)=t+O(1)$. By applying Fact 10(2) with $f(t)=1 / t$, we get

$$
\begin{aligned}
\sum_{n \leq N} \pi(n) R_{X}^{n} & =\frac{A(N)}{N}+\int_{1}^{N} \frac{A(t)}{t^{2}} d t=\frac{N+O(1)}{N}+\int_{1}^{N} \frac{t+O(1)}{t^{2}} d t \\
& =1+O\left(\frac{1}{N}\right)+\int_{1}^{N}\left(\frac{1}{t}+O\left(\frac{1}{t^{2}}\right)\right) d t \\
& =1+O\left(\frac{1}{N}\right)+\left[\log t+O\left(\frac{1}{t}\right)\right]_{1}^{N} \\
& =1+O\left(\frac{1}{N}\right)+\log N+O\left(\frac{1}{N}\right)+O(1)=\log N+O(1)+O\left(\frac{1}{N}\right),
\end{aligned}
$$

and the assertion follows.

(3) Fix an arbitrary $x$ satisfying $0<x<1$. By applying Fact 10(2) with $a_{n}=\pi(n) R_{X}^{n}$ and $f(t)=x^{t}$,

$$
\sum_{n \leq N} \pi(n) R_{X}^{n} x^{n}=A(N) x^{N}-\log x \int_{1}^{N} x^{t} A(t) d t
$$

holds. It follows from part (2) that

$\sum_{n \leq N} \pi(n) R_{X}^{n} x^{n}=\left(\log N+B_{X}+O\left(\frac{1}{N}\right)\right) x^{N}-\log x \int_{1}^{N} x^{t}\left(\log t+B_{X}+O\left(\frac{1}{t}\right)\right) d t$,

and, moreover, as $N \rightarrow \infty$,

$$
P_{X}\left(R_{X} x\right)=-\log x \int_{1}^{\infty} x^{t}\left(\log t+B_{X}+O\left(\frac{1}{t}\right)\right) d t .
$$

In order to calculate the right-hand side of this equality, for simplicity of arguments, we define the functions $I_{n}=I_{n}(x)$ :

$$
-\log x \int_{1}^{\infty} x^{t}\left(\log t+B_{X}+O\left(\frac{1}{t}\right)\right) d t=I_{1}+I_{2}+O\left(I_{3}\right)
$$


where

$$
\begin{aligned}
& I_{1}=-\log x \int_{1}^{\infty} x^{t} \log t d t \\
& I_{2}=-B_{X} \cdot \log x \int_{1}^{\infty} x^{t} d t=B_{X} \cdot x, \quad \text { and } \\
& I_{3}=-\log x \int_{1}^{\infty} \frac{x^{t}}{t} d t .
\end{aligned}
$$

First, we compute the function $I_{1}$ :

$$
I_{1}=-\int_{1}^{\infty}\left(x^{t}\right)^{\prime} \log t d t=\int_{1}^{\infty} \frac{x^{t}}{t} d t .
$$

Now we take $r=-t \log x$. Note that $\log x<0$. Then we obtain

$$
I_{1}=\int_{-\log x}^{\infty} \frac{e^{-r}}{r} d r=-\operatorname{Ei}(\log x),
$$

where $\operatorname{Ei}(z)(z \in \mathbb{C}$ and $|\operatorname{Arg}(-z)|<\pi)$ is the exponential integral

$$
-\operatorname{Ei}(-z)=\int_{z}^{\infty} \frac{e^{-r}}{r} d r
$$

(see, e.g., Equality (3.1.3) in [Lebedev 1972]). Since the function $\operatorname{Ei}(z)$ expands as

$$
\operatorname{Ei}(z)=\gamma+\log (-z)+\sum_{k=1}^{\infty} \frac{z^{k}}{k \cdot k !}
$$

(see Equality (3.1.6) in [ibid.]),

$$
I_{1}=-\gamma-\log (-\log x)+O(\log x)=-\gamma-\log (-\log x)+O(1-x) .
$$

Next we calculate the function $I_{3}$. It follows from the above result that

$$
I_{3}=-\log x \int_{1}^{\infty} \frac{x^{t}}{t} d t=(-\log x) I_{1}=O(1-x)
$$

as $x \uparrow 1$.

By combining the above results, the equality (9) is written as follows:

$$
P_{X}\left(R_{X} x\right)=-\gamma-\log (-\log x)+B_{X} x+O(1-x),
$$

and, moreover, as $x \uparrow 1$,

$$
P_{X}\left(R_{X} x\right)+\log (-\log x) \rightarrow B_{X}-\gamma .
$$

On the other hand, since

$$
\log Z_{X}\left(R_{X} x\right)=\log \frac{1}{1-x}+\log C_{X}+O(1-x)
$$


from the equality (8), as $x \uparrow 1$,

$$
\log Z_{X}\left(R_{X} x\right)+\log (-\log x)=\log \left(\frac{-\log x}{1-x}\right)+\log C_{X} \rightarrow \log C_{X} .
$$

Combining (10) with (11), we obtain

$$
\begin{aligned}
H_{X} & =\lim _{x \uparrow 1} H_{X}\left(R_{X} x\right)=\lim _{x \uparrow 1}\left(\log Z_{X}\left(R_{X} x\right)-P_{X}\left(R_{X} x\right)\right) \\
& =\lim _{x \uparrow 1}\left(\left(\log Z_{X}\left(R_{X} x\right)+\log (-\log x)\right)-\left(P_{X}\left(R_{X} x\right)+\log (-\log x)\right)\right) \\
& =\log C_{X}+\gamma-B_{X} .
\end{aligned}
$$

(4) Fix an arbitrary positive real number $N$. We define the following functions:

$$
H_{X}^{\leq N}=\sum_{n \leq N} \pi(n) \sum_{m=2}^{\infty} \frac{1}{m} R_{X}^{m n} \quad \text { and } \quad H_{X}^{>N}=\sum_{n>N} \pi(n) \sum_{m=2}^{\infty} \frac{1}{m} R_{X}^{m n} .
$$

Note that $H_{X}=H_{X}^{\leq N}+H_{X}^{>N}$. From parts (2) and (3), we obtain

$$
\sum_{n \leq N} \pi(n) R_{X}^{n}+H_{X}^{\leq N}=\log N+\gamma+\log C_{X}-H_{X}^{>N}+O\left(\frac{1}{N}\right) .
$$

Since the left-hand side of this equality is equal to

$$
\begin{aligned}
\sum_{n \leq N} \pi(n) R_{X}^{n}+H_{X}^{\leq N} & =\sum_{n \leq N} \pi(n) \sum_{m=1}^{\infty} \frac{1}{m} R_{X}^{m n} \\
& =-\sum_{n \leq N} \pi(n) \log \left(1-R_{X}^{n}\right)=-\log \left(\prod_{n \leq N}\left(1-R_{X}^{n}\right)^{\pi(n)}\right),
\end{aligned}
$$

we obtain

$$
\prod_{n \leq N}\left(1-R_{X}^{n}\right)^{\pi(n)}=\frac{e^{-\gamma}}{C_{X}} \cdot \frac{1}{N} \exp \left(H_{X}^{>N}+O\left(\frac{1}{N}\right)\right) .
$$

Since $H_{X}^{>N} \rightarrow 0$ and $1 / N \rightarrow 0$ as $N \rightarrow \infty$, the assertion follows.

Last, we compute the following example.

Example 15 (continued from Example 12). Consider the graph $X=K_{4}-$ \{one edge\}. Then

$$
H_{X}=0.25613 \ldots, \quad B_{X}=\gamma+\log C_{X}-H_{X}=-0.26933 \ldots
$$


For example, if $N=550$, then

$$
\begin{gathered}
\sum_{n \leq N} \pi(n) R_{X}^{n}-\log N=-0.26842 \ldots \approx B_{X}, \\
\prod_{n \leq N}\left(1-R_{X}^{n}\right)^{\pi(n)}=0.18447 \ldots \approx \frac{e^{-\gamma}}{C_{X}} \cdot \frac{1}{N}=0.18457 \ldots
\end{gathered}
$$

Remark 16. (See [Mertens 1874, Equation (17)], or [Hardy and Wright 2008, Theorem 428].) A number-theoretic analogue to part (3) in the preceding theorem is

$$
B_{1}=\gamma-H=\gamma+\sum_{p}\left(\log \left(1-\frac{1}{p}\right)+\frac{1}{p}\right),
$$

where $H=\sum_{n \geq 2} P(n) / n$ is a constant, and $P(s)$ is the prime zeta function.

Remark 17. We now compare parts (2)-(4) of our Theorem 14 with Theorem 1 in [Sharp 1991]. We define

$$
h_{X}:=-\log R_{X}, \quad N(P)=e^{h_{X} \ell(P)} \quad \text { and } \quad x=e^{h_{X} N} .
$$

The quantity $h_{X}$ is called the topological entropy of a flow in ergodic theory (see [Sharp 1991]), which is a constant in our setting. Note that $\ell(P) \leq N$ if and only if $N(P) \leq x$. Note that $R_{X}^{\ell(P)}=1 / N(P)$. Then our Mertens' second theorem can be rewritten as

$$
\sum_{N(P) \leq x} \frac{1}{N(P)}=\log (\log x)+B+O\left(\frac{1}{\log x}\right),
$$

where $B:=-\log h_{X}+B_{X}$, and, similarly, our Mertens' third theorem becomes

$$
\prod_{N(P) \leq x}\left(1-\frac{1}{N(P)}\right) \sim \frac{1}{C_{X} / h_{X}} \cdot \frac{e^{-\gamma}}{\log x} .
$$

In Theorem 1 in [Sharp 1991], our constant $C_{X} / h_{X}$, which is equal to a residue (up to sign) of the Ihara zeta function, corresponds with that of a dynamical zeta function for a flow.

Moreover, our Theorem 14(3) becomes

$$
B=\gamma+\log \left(C_{X} / h_{X}\right)+\sum_{[P]}\left(\log \left(1-\frac{1}{N(P)}\right)+\frac{1}{N(P)}\right) .
$$

Remark 18. Let $X=(V, E)$ be a finite, connected, non-cycle graph without degreeone vertices, and let $S=\left(V^{\prime}, E^{\prime}\right)$ be its $k$-subdivision (that is, let $S$ be the graph obtained from $X$ by adding $k$ new vertices to each edge of $X$ ) (see Examples 6.4 and 8.5 in [Terras 2011]). Then

$$
H_{X}=H_{S}, \quad C_{X}=(k+1) C_{S}, \quad \text { and } \quad B_{X}=B_{S}+\log (k+1) .
$$


This is proved as follows: note that $\Delta_{S}=(k+1) \Delta_{X}, R_{S}^{k+1}=R_{X}$, and

$$
\pi_{S}(n)= \begin{cases}\pi_{X}(n /(k+1)) & \text { if }(k+1) \mid n \\ 0 & \text { otherwise. }\end{cases}
$$

Therefore,

$$
\begin{aligned}
H_{S} & =\sum_{m \geq 2} \frac{1}{m} P_{S}\left(R_{S}^{m}\right)=\sum_{m \geq 2} \frac{1}{m} \sum_{n=1}^{\infty} \pi_{S}(n) R_{S}^{m n}=\sum_{m \geq 2} \frac{1}{m} \sum_{n=1}^{\infty} \pi_{X}(n) R_{S}^{(k+1) m n} \\
& =\sum_{m \geq 2} \frac{1}{m} \sum_{n=1}^{\infty} \pi_{X}(n) R_{X}^{m n}=\sum_{m \geq 2} \frac{1}{m} P_{X}\left(R_{X}^{m}\right)=H_{X} .
\end{aligned}
$$

Note that $v^{\prime}=v+k \epsilon, \epsilon^{\prime}=(k+1) \epsilon$, and $Z_{S}(u)=Z_{X}\left(u^{k+1}\right)$, and so

$$
\begin{aligned}
\left(1-u^{2}\right)^{\epsilon-v} f_{S}(u) & =\left(1-u^{2(k+1)}\right)^{\epsilon-v} f_{X}\left(u^{k+1}\right), \\
\left(1-R_{S}^{2}\right)^{\epsilon-v} R_{S} f_{S}^{\prime}\left(R_{S}\right) & =(k+1)\left(1-R_{X}^{2}\right)^{\epsilon-v} R_{X} f_{X}^{\prime}\left(R_{X}\right) .
\end{aligned}
$$

Therefore,

$$
(k+1) C_{S}=\frac{-(k+1)}{\left(1-R_{S}^{2}\right)^{\epsilon^{\prime}-v^{\prime}} R_{S} f_{S}^{\prime}\left(R_{S}\right)}=C_{X},
$$

and so

$$
B_{X}=\gamma+\log C_{X}-H_{X}=\gamma+\log C_{S}-H_{S}+\log (k+1)=B_{S}+\log (k+1) .
$$

\section{Acknowledgements}

Saito would like to thank Professor Toyokazu Hiramatsu for his encouragement and thoughtful suggestions. The authors thank the referee for his or her valuable comments and careful review of this paper.

\section{References}

[Apostol 1974] T. M. Apostol, Mathematical analysis, 2nd ed., Addison-Wesley, Reading, MA, 1974. MR 49 \#9123 Zbl 0309.26002

[Bass 1992] H. Bass, “The Ihara-Selberg zeta function of a tree lattice”, Internat. J. Math. 3:6 (1992), 717-797. MR 94a:11072 Zbl 0767.11025

[Fröberg 1968] C.-E. Fröberg, "On the prime zeta function”, Nordisk Tidskr. Informationsbehandling (BIT) 8 (1968), 187-202. MR 38 \#4421 Zbl 0167.04201

[Glaisher 1891] J. W. L. Glaisher, "On the sums of the inverse powers of the prime numbers", Quart. J. Pure. Appl. Math. 25 (1891), 347-362. JFM 23.0275.02

[Hardy and Wright 2008] G. H. Hardy and E. M. Wright, An introduction to the theory of numbers, 6th ed., Oxford University Press, 2008. Revised by D. R. Heath-Brown and J. H. Silverman. MR 2009i:11001 Zbl 1159.11001 
[Hashimoto 1989] K.-I. Hashimoto, "Zeta functions of finite graphs and representations of $p$ adic groups", pp. 211-280 in Automorphic forms and geometry of arithmetic varieties, edited by K.-I. Hashimoto and Y. Namikawa, Adv. Stud. Pure Math. 15, Academic Press, Boston, 1989. MR 91i:11057 Zbl 0709.22005

[Horton et al. 2006] M. D. Horton, H. M. Stark, and A. A. Terras, "What are zeta functions of graphs and what are they good for?", pp. 173-189 in Quantum graphs and their applications, edited by G. Berkolaiko et al., Contemp. Math. 415, Amer. Math. Soc., Providence, RI, 2006. MR 2007i:05088 Zbl 1222.11109

[Ihara 1966] Y. Ihara, "On discrete subgroups of the two by two projective linear group over $\mathfrak{p}$-adic fields", J. Math. Soc. Japan 18 (1966), 219-235. MR 36 \#6511 Zbl 0158.27702

[Jameson 2003] G. J. O. Jameson, The prime number theorem, London Mathematical Society Student Texts 53, Cambridge University Press, 2003. MR 2004c:11002 Zbl 1033.11001

[Korevaar 2002] J. Korevaar, "A century of complex Tauberian theory", Bull. Amer. Math. Soc. (N.S.) 39:4 (2002), 475-531. MR 2003g:40004 Zbl 1001.40007

[Kotani and Sunada 2000] M. Kotani and T. Sunada, "Zeta functions of finite graphs", J. Math. Sci. Univ. Tokyo 7:1 (2000), 7-25. MR 2001f:68110 Zbl 0978.05051

[Landau and Walfisz 1920] E. Landau and A. Walfisz, "Über die Nichtfortsetzbarkeit einiger durch Dirichletsche Reihen definierter Funktionen”, Rend. Circ. Mat. Palermo 44 (1920), 82-86. JFM 47.0287.02

[Lebedev 1972] N. N. Lebedev, Special functions and their applications, 2nd ed., Dover, New York, 1972. MR 50 \#2568 Zbl 0271.33001

[Mertens 1874] F. Mertens, "Ein Beitrag zur analytischen Zahlentheorie", J. Reine Angew. Math. 78 (1874), 46-63. JFM 06.0116.01

[Northshield 1998] S. Northshield, "A note on the zeta function of a graph", J. Combin. Theory Ser. B 74:2 (1998), 408-410. MR 99g:05106 Zbl 1027.05048

[Parry 1983] W. Parry, "An analogue of the prime number theorem for closed orbits of shifts of finite type and their suspensions", Israel J. Math. 45:1 (1983), 41-52. MR 85c:58089 Zbl 0552.28020

[Parry and Pollicott 1983] W. Parry and M. Pollicott, "An analogue of the prime number theorem for closed orbits of Axiom A flows", Ann. of Math. (2) 118:3 (1983), 573-591. MR 85i:58105 Zbl 0537.58038

[Sharp 1991] R. Sharp, "An analogue of Mertens' theorem for closed orbits of Axiom A flows", Bol. Soc. Brasil. Mat. (N.S.) 21:2 (1991), 205-229. MR 93a:58142 Zbl 0761.58041

[Stark and Terras 1996] H. M. Stark and A. A. Terras, "Zeta functions of finite graphs and coverings", Adv. Math. 121:1 (1996), 124-165. MR 98b:11094 Zbl 0874.11064

[Storm 2010] C. Storm, "An infinite family of graphs with the same Ihara zeta function", Electron. J. Combin. 17:1 (2010), Research Paper 82. MR 2011g:05156 Zbl 1215.05096

[Terras 2011] A. A. Terras, Zeta functions of graphs: a stroll through the garden, Cambridge Studies in Advanced Mathematics 128, Cambridge University Press, 2011. MR 2012d:05016 Zbl 1206.05003

[Titchmarsh 1958] E. C. Titchmarsh, The theory of functions, 2nd ed., Oxford University Press, 1958. MR 3155290 Zbl 0336.30001

[Titchmarsh 1986] E. C. Titchmarsh, The theory of the Riemann zeta-function, 2nd ed., Clarendon, New York, 1986. MR 88c:11049 Zbl 0601.10026

Received October 3, 2013. Revised May 29, 2014. 
TAKEHIRO HASEGAWA

SHIGA UNIVERSITY

OTSU

SHIGA 520-0862

JAPAN

thasegawa3141592@yahoo.co.jp

SEIKEN SAITO

WASEDA UNIVERSITY

SHINJUKU

TOKYO 169-8050

JAPAN

seiken.saitou@gmail.com 


\title{
PACIFIC JOURNAL OF MATHEMATICS
}

\author{
msp.org/pjm
}

Founded in 1951 by E. F. Beckenbach (1906-1982) and F. Wolf (1904-1989)

\section{EDITORS}

Don Blasius (Managing Editor)

Department of Mathematics

University of California

Los Angeles, CA 90095-1555

blasius@math.ucla.edu

\author{
Paul Balmer \\ Department of Mathematics \\ University of California \\ Los Angeles, CA 90095-1555 \\ balmer@math.ucla.edu \\ Robert Finn \\ Department of Mathematics \\ Stanford University \\ Stanford, CA 94305-2125 \\ finn@math.stanford.edu \\ Sorin Popa \\ Department of Mathematics \\ University of California \\ Los Angeles, CA 90095-1555 \\ popa@math.ucla.edu
}

\author{
Vyjayanthi Chari \\ Department of Mathematics \\ University of California \\ Riverside, CA 92521-0135 \\ chari@math.ucr.edu \\ Kefeng Liu \\ Department of Mathematics \\ University of California \\ Los Angeles, CA 90095-1555 \\ liu@math.ucla.edu \\ Jie Qing \\ Department of Mathematics \\ University of California \\ Santa Cruz, CA 95064 \\ qing@ cats.ucsc.edu
}

\section{PRODUCTION}

Silvio Levy, Scientific Editor, production@msp.org

\section{SUPPORTING INSTITUTIONS}

ACADEMIA SINICA, TAIPEI

CALIFORNIA INST. OF TECHNOLOGY

INST. DE MATEMÁTICA PURA E APLICADA

KEIO UNIVERSITY

MATH. SCIENCES RESEARCH INSTITUTE

NEW MEXICO STATE UNIV.

OREGON STATE UNIV.

\author{
STANFORD UNIVERSITY \\ UNIV. OF BRITISH COLUMBIA \\ UNIV. OF CALIFORNIA, BERKELEY \\ UNIV. OF CALIFORNIA, DAVIS \\ UNIV. OF CALIFORNIA, LOS ANGELES \\ UNIV. OF CALIFORNIA, RIVERSIDE \\ UNIV. OF CALIFORNIA, SAN DIEGO \\ UNIV. OF CALIF., SANTA BARBARA
}

\author{
Daryl Cooper \\ Department of Mathematics \\ University of California \\ Santa Barbara, CA 93106-3080 \\ cooper@math.ucsb.edu \\ Jiang-Hua Lu \\ Department of Mathematics \\ The University of Hong Kong \\ Pokfulam Rd., Hong Kong \\ jhlu@maths.hku.hk \\ Paul Yang \\ Department of Mathematics \\ Princeton University \\ Princeton NJ 08544-1000 \\ yang@math.princeton.edu
}

These supporting institutions contribute to the cost of publication of this Journal, but they are not owners or publishers and have no responsibility for its contents or policies.

See inside back cover or msp.org/pjm for submission instructions.

The subscription price for 2015 is US \$420/year for the electronic version, and \$570/year for print and electronic.

Subscriptions, requests for back issues and changes of subscribers address should be sent to Pacific Journal of Mathematics, P.O. Box 4163, Berkeley, CA 94704-0163, U.S.A. The Pacific Journal of Mathematics is indexed by Mathematical Reviews, Zentralblatt MATH, PASCAL CNRS Index, Referativnyi Zhurnal, Current Mathematical Publications and Web of Knowledge (Science Citation Index).

The Pacific Journal of Mathematics (ISSN 0030-8730) at the University of California, c/o Department of Mathematics, 798 Evans Hall \#3840, Berkeley, CA 94720-3840, is published twelve times a year. Periodical rate postage paid at Berkeley, CA 94704, and additional mailing offices. POSTMASTER: send address changes to Pacific Journal of Mathematics, P.O. Box 4163, Berkeley, CA 94704-0163.

PJM peer review and production are managed by EditFLOW ${ }^{\circledR}$ from Mathematical Sciences Publishers.

\section{PUBLISHED BY}

\section{mathematical sciences publishers \\ nonprofit scientific publishing}

http://msp.org/

(C) 2015 Mathematical Sciences Publishers 


\section{PACIFIC JOURNAL OF MATHEMATICS}

Volume $273 \quad$ No. $1 \quad$ January 2015

Maximal estimates for Schrödinger equations with inverse-square potential 1

ChangXing MiaO, JUNYong Zhang and JiQiang Zheng

Vassiliev Invariants of Virtual Legendrian Knots

PATRICIA CAHN and ASA LEVI

Some results on the generic vanishing of Koszul cohomology via

deformation theory

JIE WANG

Conformal metrics with constant curvature one and finitely many conical

singularities on compact Riemann surfaces

QING Chen, WeI WANG, Yingyi Wu and BIN XU

$\mathbb{Q}$-bases of the Néron-Severi groups of certain elliptic surfaces

MASAMICHI KURODA

On a prime zeta function of a graph

TAKehiro Hasegawa and SEIKEN SAIto

On Whittaker modules for a Lie algebra arising from the 2-dimensional torus 147

SHaObin TAN, QING WANG and ChENGKANG XU

Fréchet quantum supergroups

AXEL DE GOURSAC

Generators of the Gauss-Picard modular group in three complex dimensions

BaoHua Xie, JieYan Wang and YuePing Jiang

Complete characterization of isolated homogeneous hypersurface singularities

STEPHEN YAu and HuAiQING ZuO

A theorem of Mœglin and Waldspurger for covering groups

SHIV PRAKASH PATEL

Spanning trees and random walks on weighted graphs 